\title{
Ototoxicité des aminoglycosides
}

Les antibiotiques de la famille des aminoglycosides ont une ototoxicité secondaire à leur fixation avec une forte affinité sur la membrane des cellules ciliées de la cochlée; les lésions progressent de la base vers l'apex et touchent successivement les cellules ciliées externes, puis internes. Des moyens modernes d'exploration électroacoustique des cellules ciliées externes permettent de détecter les lésions à un stade très précoce, où elles sont encore réversibles.

\section{Patrice Tran Ba Huy Professeur à la faculté de médecine de Paris. \\ ORL des hôpitaux de. Paris}

\section{RÉFÉRENCES}

1. Hawkins JE. Drug ototoxicity. In : Keidel WD, Neff WD, eds. Handbook of Sensory Physiology. Berlin, Heidelberg, New York : Springer, 1976, 707-48.

2. Hawkins JE. Cochlear signs of streptomycin intoxication. Acta Otolaryngol (Stockh) 1977 ; $84: 65-71$.

3. Wersäll J, Flock A. Suppression and restoration of the microphonic output from the lateral line organ after local application of streptomycin. Life Sci 1964 ; 3 : 1151-5.

4. Galambos R, Hecox K. Clinical applications of the brainstem auditory evoked potentials. In : Desmedt J, ed. Auditory evoked potentials in man. Progress in Clinical Neurophysiology. Bâle : Karger, 1977 ; 1-19.

5. Voldrich L. The kinetics of streptomycin kanamycin and neomycin in the inner ear. Acto Otolaryngol (Stockh) 1965 ; 60 : 243-8.

\section{ADRESSE}

P. Tran Ba Huy : chaire de clinique ORL hôpital Lariboisière, faculté Lariboisière-StLouis, et Inserm U. 251, faculté X. Bichat, Paris, France.

\section{TIRÉS A PART}

P. Tran Ba Huy : chaire de clinique ORL hôpital Lariboisière, 2, rue Ambroise-Paré, 75010 Paris, France. a publication en 1945 par Hinshaw et Feldmann d'accidents cochléovestibulaires chez des malades tuberculeux traités par un nouvel antibiotique, la streptomycine, a constitué un véritable tournant dans l'histoire de l'ototoxicité. En effet, cette remarquable spécificité toxique pour l'oreille, régulièrement confirmée depuis pour tous les nouveaux membres de cette famille médicamenteuse, les aminoglycosides, et bientôt complétée par la constatation de leur non moins remarquable néphrotoxicité, a suscité un nombre considérable de travaux. Ces travaux ont eu pour but à la fois d'élucider les mécanismes de ces accidents afin de tenter de les prévenir et d'étudier la physiologie cochléo-vestibulaire elle-même, puisque ces substances agissent sélectivement sur les structures neuro-sensorielles. Quoi qu'il en soit, ces premiers travaux histologiques, électrophysiologiques et pharmacocinétiques avaient permis d'établir un schéma pathogénique relativement simple de l'ototoxicité. Ce schéma prévalût jusqu'à ces dernières années mais s'est trouvé sensiblement modifié par l'apparition de nouvelles techniques de dosages et par les progrès de la physiologie cochléaire. L'introduction de nouvelles modalités d'administration de ces anti- biotiques, toujours très efficaces dans le traitement des infections sévères, jointe aux progrès de la réanimation qui permettent de surmonter l'éventuelle atteinte rénale, conferrent à l'heure actuelle un regain d'intérêt aux études conduites sur l'ototoxicité. Le but de cet article est de retracer les principales étapes de ces études sur la physiopathologie de l'ototoxicité.

\section{Histologie}

Les études histologiques furent les premières menées ; elles permirent de rapporter à l'oreille interne (figures 1 et 2) les accidents initialement attribués à une névrite de la huitième paire crânienne et d'en décrire les remarquables caractéristiques lésionnelles [1]. L'atteinte affecte essentiellement les structures neuro-sensorielles, et s'étend, dans la cochlée, selon une direction à la fois baso-apicale et radiale : sont ainsi successivement touchées les cellules ciliées externes du premier, deuxième et troisième rang puis les cellules ciliées internes et ce, de la spire basale à la spire apicale. La raison de cette étonnante progression lésionnelle n'est pas encore clairement élucidée. Cette évolution de l'atteinte des cellules sensorielles de la base vers l'apex permet de comprendre pourquoi la surdité observée en cas d'intoxication par 


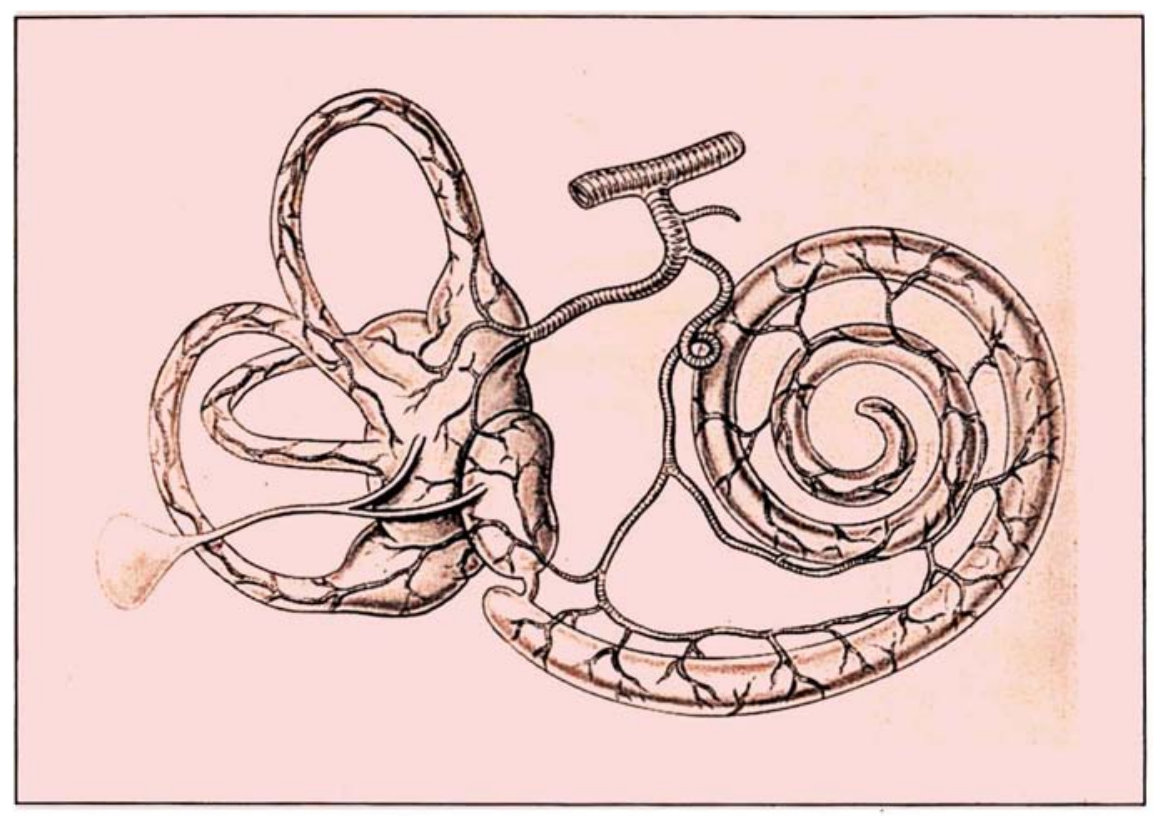

Figure 1. Schéma du labyrinthe membraneux et de sa vascuiarisation. Le labyrinthe comprend une partie antérieure (à droite) constituée par le canal cochléaire enroulé en limaçon et une partie postérieure (à gauche) constituée par le saccule et l'utricule. Dans ce dernier, s'ouvrent les trois canaux semi-circulaires. Du saccule et de l'utricule partent les deux branches constitutives de l'aqueduc du vestibule qui mène au sac endolymphatique là l'extrême gauche). La vascularisation est assurée par l'artère auditive interne, branche de l'artère cérébelleuse. Elle est de type terminal.

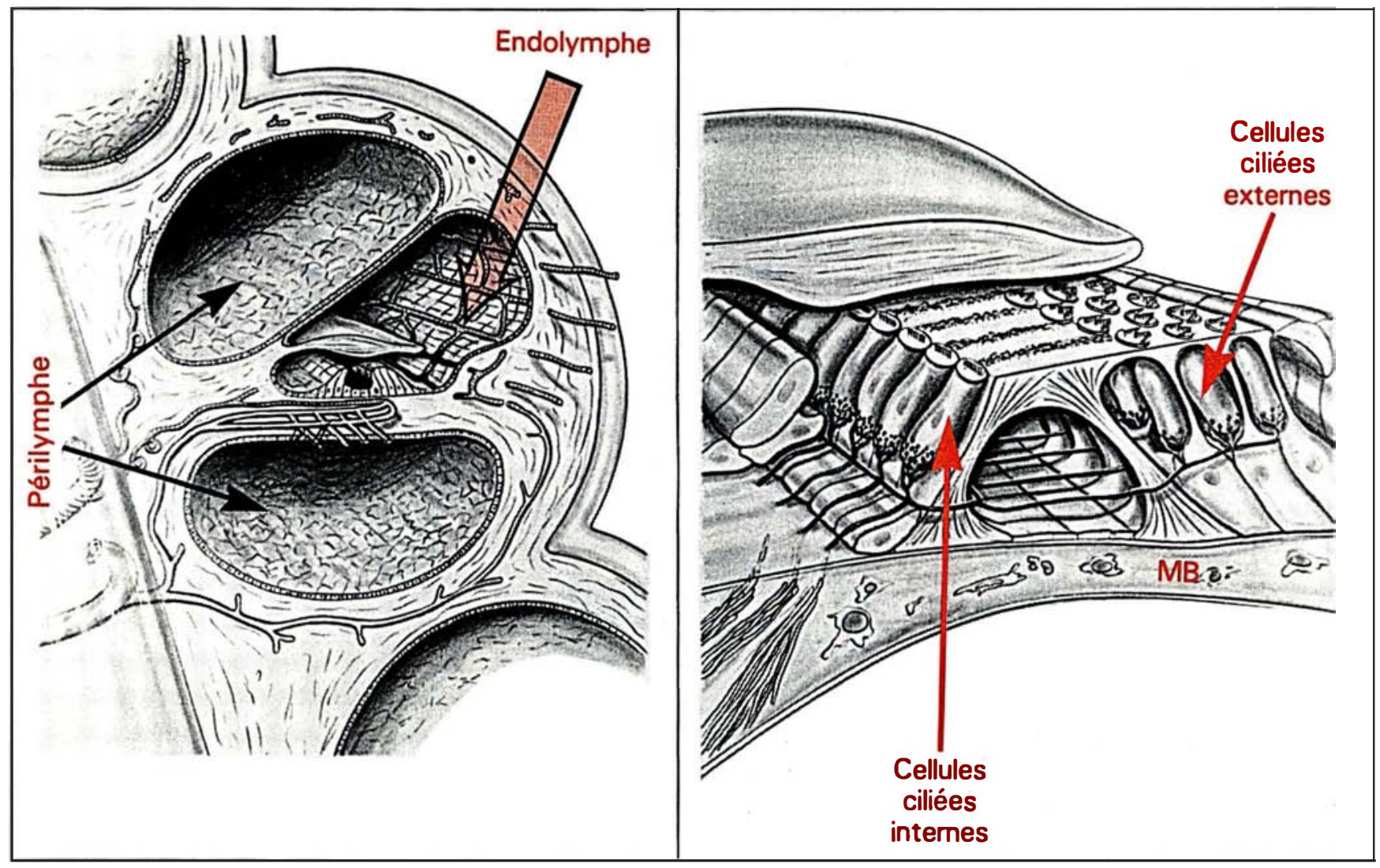

Figure 2. Schéma du canal cochléaire. A gauche : section du canal montrant les deux compartiments périlymphatiques (en haut et en bas) (flèches noires) entourant le compartiment endolymphatique à droite et au milieu (flèche rosel. Notez que la vascularisation du canal cochléaire est constituée d'un réseau radial externe vascularisant surtout la strie vasculaire et d'un réseau radial interne cheminant sous l'organe de Corti sans le vasculariser directement. Tout produit devant atteindre l'organe sensoriel doit donc nécessairement diffuser à travers les liquides labyrinthiques. A droite, détail de l'organe de Corti montrant le tunnel de Corti surmonté à gauche de la rangée de cellules ciliées internes (flèche rouge) et à droite des trois rangées de cellules ciliées externes (flèche rouge); $M B=$ membrane basilaire. $\mathrm{m} / \mathrm{s} n^{\circ} 1$ vol. 4, janvier 88 
les aminoglycosides progresse des fréquences aiguës vers les fréquences graves*. Sur le plan cellulaire, les étapes de l'intoxication sont marquées par une altération rapidement progressive des stéréocils, une prolifération du réticulum endoplasmique, une dégénérescence mitochondriale, une vacuolisation cellulaire, un œdème nucléaire puis une désintégration cellulaire. L'essentiel est d'observer que les stigmates de l'ototoxicité different sensiblement de ceux décrits dans le tube rénal. En effet, les lésions rénales dues à l'administration d'aminosides sont caractérisées par l'accumulation de phospholipides dans les lysosomes des cellules tubulaires proximales.

\section{Électrophysiologie}

Entreprises dès 1950, les études électrophysiologiques montrèrent que l'administration d'aminosides altérait précocément les potentiels microphoniques cochléaires** [2]. Ceci correspondait bien aux constatations histologiques puisque les cellules responsables de ces potentiels sont précisément les cellules ciliées externes (figure 2). Fait essentiel, ces potentiels pouvaient être altérés de façon réversible ou non selon la concentration et la durée d'exposition à la drogue ainsi que la richesse en potassium du milieu [3]. Cette observation rendait bien compte de la réversibilité possible de certaines manifestations ototoxiques cliniques si l'administration médicamenteuse était suspendue dès les premiers signes. Puis, des altérations précoces des potentiels de sommation ${ }^{* * *}$ et d'action ${ }^{* * * *}$ du nerf auditif, et des potentiels évoqués auditifs du tronc cérébral furent observées [4]. La caractéristique essentielle de toutes ces altérations était qu'elles précédaient les altérations morphologiques, d'où l'intérêt majeur des techniques d'électrocochléographie et de recueil de potentiels évoqués auditifs (figure 3) introduites dans les années 70 dans la surveillance des patients traités par les aminoglycosides. Le mécanisme d'appari-

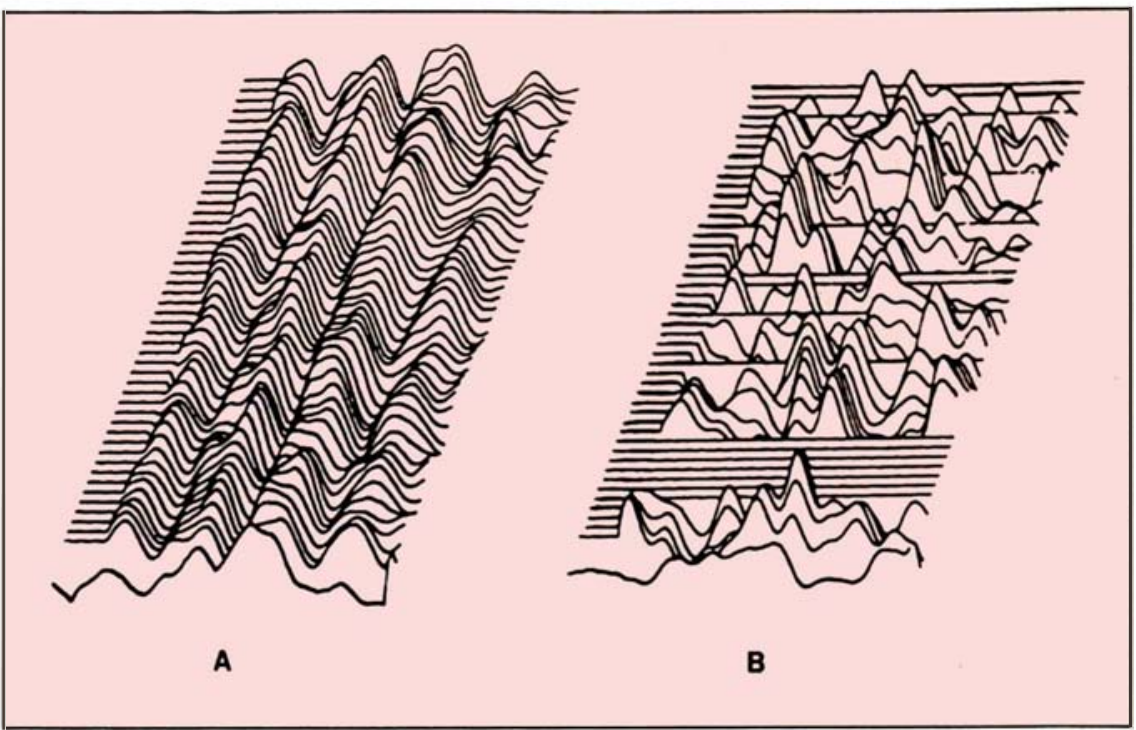

Figure 3. Tracés de potentiels évoqués auditifs du tronc cérébral. A. tracé normal visualisant bien les cinq ondes. B. tracé désorganisé témoignant d'une atteinte endocochléaire, telle qu'on peut en observer au cours d'intoxications aminosidiques.

trophysiologiques, incomplètement expliqué, pourrait être similaire à celui par lequel ces mêmes aminosides bloquent la neurotransmission au niveau de la plaque motrice : l'antibiotique interférerait avec le calcium à la terminaison nerveuse bloquant ainsi la libération pré-synaptique du neurotransmetteur.

\section{Pharmacocinétique}

La pharmacocinétique des aminosides fut d'abord étudiée dans les liquides labyrinthiques $[5,6]$. Ceux-ci représentent, en effet, la voie d'accès obligatoire aux structures neuro-sensorielles non vascularisées de l'oreille interne. Quel que soit le protocole expérimental adopté, toutes ces études ont démontré que la demi-vie périlymphatique de l'aminoglycoside considéré était nettement supérieure à la demi-vie plasmatique. De cette observation naquit la théorie dite de "l'accumulation " [7] selon laquelle l'ototoxicité des aminosides était directement liée à leur accumulation dans la périlymphe : plus la concentration périlymphatique d'un aminoglycoside et sa demi-vie étaient élevées, plus il était ototoxique. Ainsi, l'idée s'était-elle répandue que l'oreille interne agissait comme un piège spécifique vis-à-vis des aminoglycosides. Le mécanisme de cette action délétère était supposé s'exercer, comme au niveau bactérien, par le biais d'une altération de la sous-unité $30 \mathrm{~S}$ ribosomiale perturbant la synthèse protéique de la cellule sensorielle.

Telle était donc la conception classique, essentiellement basée sur des considérations pharmacocinétiques, qui, durant plusieurs

\footnotetext{
- Il existe une tonotropie cochléaire réalisant une gamme de fréquences allant de la base vers l'apex. Cette spécialisation fréquentielle explique que les cellules sensorielles de la base soient sensibles aux fréquences aigués alors que celles de l'apex le sont aux fréquences graves.

- Potentiel de récepteur traduisant la réponse électrophysiologique de l'organe de Corti à toule stimulation acoustique. Il reproduit électriquement, selon un systeme analogique, le son qui lui a donné naissance. Il témoigne fidèlement de l'activité des cellules sensorielles.

- Potentiel de récepteur traduisant également l'activité des cellules ciliées et se superposant au potentiel microphonique.

-... Il reflète l'activité bioélectrique propagée sous forme d'influx, le long du nerf auditif en direction des centres.
} 


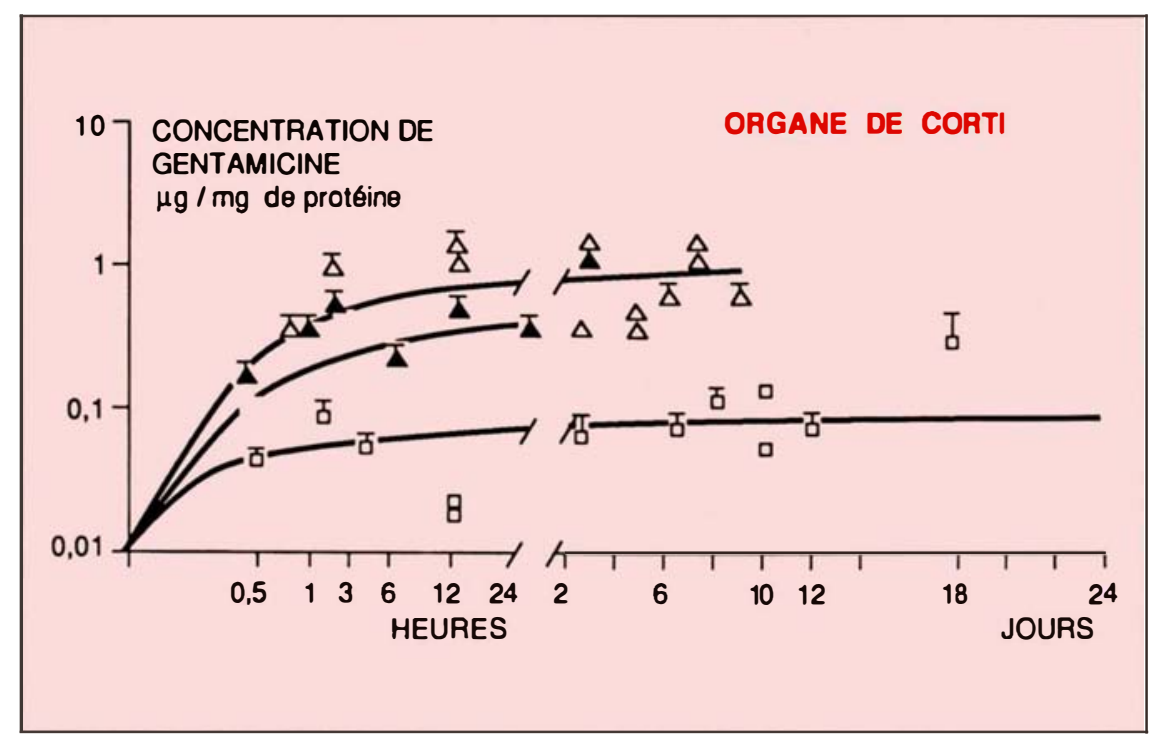

Figure 4. Pharmacocinétique de la gentamicine dans l'organe de Corti en fonction de différents taux de perfusion $11,5 \mu \mathrm{g} / \mathrm{min}=\square ; 15 \mu \mathrm{g} / \mathrm{min}$ $=\Delta ; 150 \mu \mathrm{g} / \mathrm{min}=\Delta ;$ le symbole $T=S E M$, standard error of mean). Un plateau est atteint dès la $6^{\circ}$ heure.

années, a permis d'expliquer la survenue des phénomènes ototoxiques. Cette conception s'est trouvée sensiblement nuancée par des études récentes utilisant des méthodes de dosages plus sensibles et menées sur la base d'une analyse critique des méthodologies précédentes. En effet, les précédents travaux n'avaient porté que sur la périlymphe, n'envisageant donc qu'un seul des deux liquides labyrinthiques et négligeant surtout la cible finale que sont les structures neurosensorielles ellesmêmes. De plus, des doses considérables d'antibiotiques, très largement supérieures aux doses thérapeutiques, avaient été administrées afin de contrebalancer la faible sensibilité des méthodes de dosage de l'époque.

Des études pharmacocinétiques menées conjointement sur la périlymphe, l'endolymphe, et l'organe de Corti (figure 2, page 37) ont pu préciser la cinétique de captation et de relargage d'un aminoglycoside de référence, la gentamicine, dosé par méthodes radio-immunologiques $[8,9]$. La captation de l'antibiotique par l'organe de Corti apparaît remarquable par sa rapidité et sa satu$\mathrm{m} / \mathrm{s} n^{\circ} 1$ vol. 4, janvier 88
Cette étude fournit également un élément de réponse à la question des dangers respectifs de l'administration continue ou discontinue. Dans nos conditions expérimentales, la concentration tissulaire atteinte dans l'organe de Corti après une injection intramusculaire unique de $100 \mathrm{mg} / \mathrm{kg}$ est environ 15 fois inférieure à celle obtenue après perfusion d'une dose équivalente à $15 \mathrm{mg} / \mathrm{kg}$ en trois heures. Un tel résultat s'inscrit manifestement en faveur des administrations quotidiennes discontinues, si possible uniques, entraînant un pic sérique élevé, mais bref.

Ces données tissulaires soulignent bien le rôle des liquides labyrinthiques qui représentent, comme nous l'avons vu plus haut, la voie d'emprunt imposée et influencent directement les cinétiques tissulaires. La périlymphe se comporte comme un compartiment profond que l'aminoside ne pénètre et ne quitte que lentement en raison de la présence d'une barrière hémato-labyrinthique analogue à la barrière hémato-cérébrale [10]. La demi-vie d'élimination, sensiblement identique quel que soit l'aminoside considéré, y est d'environ trois heures. Les concentrations atteintes sont corrélées aux concentrations plasmatiques dont elles représentent 10 à $15 \%$. Ceci infirme deux notions classiques : d'une part l'existence d'un seuil plasmatique toxique au-delà duquel l'aminoside est capable de pénétrer dans les liquides labyrinthiques [7] et, d'autre part, la présence d'une accumulation de l'antibiotique dans la périlymphe $[5,7]$. L'endolymphe se définit comme un compartiment encore plus profond que la périlymphe. L'antibiotique n'y est détectable qu'après plusieurs heures, et, une fois entré, y séjourne si longtemps que la détermination précise de sa demi-vie d'élimination reste techniquement impossible à établir [11]. Le rôle respectif de ces deux liquides dans l'apparition des phénomènes ototoxiques est suggéré par la comparaison des cinétiques de pénétration tissulaire et liquidienne. La 


\section{RÉFÉRENCES}

6. Vrabec DP, Cody DT, Ulrich JA. A study of the relative concentrations of antibiotics in the blood, spinal fluid and perilymph in animals. Ann Otol Rhinol Laryngol 1965. ; 74 : 688-705.

7. Stupp HF. Untersuchung der Antibiotikaspiegel in den Innenohrflüssigkeiten und ihre Bedeutung für die spezifische Ototoxizität der aminoglykosidantibiotika. Acta Otolagngol (Stockh) 1970 ; 262 (suppl) : 1-85.

8. Tran Ba Huy P, Manuel C, Meulemans A, Sterkers O, Amiel C. Pharmacokinetics of gentamicin in perilymph and endolymph of the rat as determined by radioimmunoassay. $J$ Infact $D$ is $1981 ; 143: 476-86$.

9. Tran Ba Huy P, Bernard P, Schacht J. Kinetics of gentamicin uptake and release in the rat. Comparison of inner ear tissues and fluids with other organs. J Clin Inoest 1986 ; 77 : 1492-1500.

10. Sterkers O, Ferrary E, Saumon G, Amiel C. $\mathrm{Na}$ and nonelectrolyte entry into the inner ear fluids of the rat. Am J Physiol 253 (Renal Fluid Electrolytes Physiol. 22) 1987 (sous presse).

11. Tran Ba Huy $P$, Meulemans A, Wassef $M$, Manuel C, Sterkers O. Gentamicin persistence in rat endolymph and perilymph after a two-day constant infusion. Antimicrob Agents Chemother $1983 ; 23: 344-6$.

12. Konishi T. Effects of local application of ototoxic antibiotics on cochlear potentials in guinea pigs. Acta Otolaryngol (Stockh) 1979 ; 88 : 41-50.

13. Tran Ba Huy P, Deffrennes D. Evidence for specific binding sites of gentamicin in the organ of Corti and in the vestibular sensory structures. Abstract of the X ARO meeting. Clearwater Beach : FRO, 1987.

14. Sastrasinh M, Knauss TC, Weinberg JM, Humes HD. Identification of the aminoglycoside binding site in rat renal brush border membranes. J Pharmacol Exp Ther 1982 ; 222 : 350-8.

15. Brasseur R, Laurent G, Ruysschaert MM, Tulkens P. Interactions of aminoglycoside antibiotics with negatively charged lipid layers. Biochem Pharmacol 1984 ; 33 : 629-37.

16. Schacht J, Weiner N. Aminoglycosideinduced hearing loss : a molecular hypothesis. ORL $1986 ; 48$ : 116-23.

17. Papahadjopoulos D, Jacobson $\mathrm{K}$, Nir S, Isac $T$. Phase transitions in phospholipid vesicles. Fluorescence polarization and permeability measurements concerning the effect of temperature and cholesterol. Biochim Biophys Acta 1973 ; 311 : 330-48.

18. Takada A, Schacht J. Calcium antagonism and reversibility of gentarnicin-induced loss microphonics in the guinea pig. Hear Res 1982 ; 8 : 179-86.

19. Brownell WE, Bader CR, Bertrand D, De Ribaupierre Y. Evoked mechanical responses of isolated cochlear outer hair cells. Science 1985 ; 227 : 194-6.

20. Flock A, Flock B, Ulfendahl M. Mechanisms of movement in outer hair cells and a possible structural basis. Arch Otorkinolaryngol 1986 ; 243 : 83-90.

21. Kemp DT. Stimulated acoustic emission from within the human auditory system J Acoust périlymphe, qui baigne le pôle inférieur et les faces latérales des cellules ciliées, représente sans doute la voie d'accès privilégiée pour l'antibiotique aux structures sensorielles puisque le plateau tissulaire est obtenu alors que le produit, présent dans la périlymphe, n'est toujours pas détectable dans l'endolymphe. Ceci ne signifie pas pour autant que ce dernier liquide, qui baigne les stéréocils situés au pôle apical des cellules sensorielles et où se maintiennent de façon prolongée des concentrations très significatives de l'antibiotique, ne puisse jouer un rôle déterminant dans le déclenchement des phénomènes ototoxiques, comme l'avaient déjà suggéré certaines expériences électrophysiologiques [12]. Il n'est pas clairement établi si les cellules de l'organe de Corti sont d'abord atteintes par leur pôle basal ou apical. Cependant, dans tous les cas, périlymphe et endolymphe constituent un compartiment intermédiaire, amortissant les pics sériques mais maintenant des concentrations prolongées d'antibiotiques au contact des structures sensorielles. Au terme de ces études, trois facteurs caractérisent la pharmacocinétique des aminosides dans l'oreille interne, à savoir la rapidité de captation, la précocité de la saturation, et l'exposition prolongée des tissus au produit.

A l'échelon membranaire, la fixation de gentamicine marquée a pu être étudiée sur des homogénats d'organes de Corti et de macules vestibulaires (utricule et saccule) [13]. Les résultats confirment que la captation de l'antibiotique est rapide et saturable, un plateau de fixation est obtenu précocément dès la cinquième minute. $\mathrm{La}$ fixation est spécifique. Les $K_{D}$ (constante de dissociation) déterminés après analyse de Scatchard sont d'environ $10^{-6} \mathrm{M}$ pour l'organe de Corti et de $10^{-7} \mathrm{M}$ pour les macules vestibulaires. Ces valeurs, inférieures à celles rapportées dans le cortex rénal [14], suggèrent une plus forte affinité de la gentamicine pour l'oreille interne que pour le rein. Le plus faible $K_{D}$ déterminé dans les structures vestibulaires pourrait rendre compte de la toxicité de la gentamicine, plus marquée pour le vestibule que pour la cochlée. De la même façon, la toxicité préférentielle de certains aminosides pour les structures auditives ou vestibulaires pourrait s'expliquer par des affinités différentes pour les deux types de tissus. Ainsi la streptomycine, très toxique pour le vestibule mais peu pour la cochlée, déplace-t-elle beaucoup moins facilement la gentamicine de son site de liaison cochléaire que la néomycine dont la toxicité cochléaire majeure est bien établie. Dans cette échelle d'affinité, certaines discordances sont cependant observées, comme avec la nétilmicine, peu toxique pour la cochlée malgré une forte capacité de déplacement. La raison n'est donc pas encore totalement élucidée de la surprenante toxicité différentielle observée pour des structures anatomiquement si proches, de la part de molécules biochimiquement si voisines.

\section{La membrane, cible de l'ototoxicité}

La fixation semble fonction de l'affinité entre les pôles cationiques de la molécule médicamenteuse basique et les sites de liaison membranaire anioniques représentés par certains de ses composants phospholipidiques acides. Le degré de liaison dépend en effet, non seulement des forces hydrophobes mais aussi et surtout d'une interaction électrostatique entre groupes aminés et phosphorés [15]. Pour certains auteurs [16], cette étape membranaire serait multiséquentielle. Dans un premier temps, la fixation serait réversible et compétitive avec le calcium; dans un second temps, la captation deviendrait irréversible, par incorporation définitive de l'antibiotique à la membrane. L'antibiotique pénétrerait dans la cellule selon un processus actif pour former un complexe indissociable avec des phospholipides situés à la face interne de la membrane. En fait, cette conception biséquentielle de la fixation membranaire de l'anti- 
biotique est discutée. La réalité du transport actif, notamment, paraît loin d'être établie. En effet, nos résultats montrent que la présence de dinitrophénol, poison du métabolisme mitochondrial, n'affecte en rien le degré de captation. De plus, celle-ci est plus importante à $4^{\circ} \mathrm{C}$ qu'à $37^{\circ} \mathrm{C}$, ce qui, d'une part, confirme l'absence de tout mécanisme de transport actif et, d'autre part, suggère l'existence d'une transition thermotropique de phase des phospholipides membranaires* [17], offrant à basse température une configuration moléculaire d'accueil plus favorable à l'antibiotique.

Quel qu'en soit le mécanisme, il reste que cette étape membranaire paraît déterminante dans le développement des phénomènes ototoxiques. L'hypothèse d'une interférence de l'antibiotique sur la synthèse protéique ne saurait rendre compte des phénomènes électrophysiologiques aigus et réversibles observés après administration d'aminosides [18]. Comme dans le rein, la fixation de l'aminoside sur les sites de liaisons phospholipidiques permet d'expliquer l'inhibition de certaines fonctions dépendantes $\mathrm{du}^{\mathrm{Ca}^{+}+}$essentielles à la transduction mécano-électrique de l'information acoustique, puis l'altération de la perméabilité cellulaire conduisant à des flux ioniques létaux.

\section{Les cellules ciliées externes en première ligne}

Telles sont les données actuelles dont dispose le clinicien pour comprendre les mécanismes de survenue des accidents ototoxiques. Elles revêtent aujourd'hui un intérêt tout particulier avec l'introduction de nouveaux procédés d'explorations fonctionnelles permettant d'interroger de façon très sélective les cellules ciliées

\footnotetext{
* Il s'agit de le modification constitutive de la membrane sous l'effel d'une variation de température.

- Structure supportant l'organe de Corti et jouant, grâce à ses propriétés physiques, un rôle de résonnateur et d'analyseur de fréquence.

$\mathrm{m} / \mathrm{s} n^{\circ} 1$ vol. 4 , janvier 88
}

externes (CCE), premières touchées par ces antibiotiques. Un ensemble de travaux récents a, en effet, permis d'établir qu'à la différence de leurs homologues internes qui assurent de façon passive la transduction mécano-électrique, les CCE jouent un rôle dans la micromécanique cochléaire [19]. Par leur contraction, modulée par le système nerveux central par le biais d'une innervation efférente massive et mettant en jeu un système de protéines contractiles [20], ces véritables cellules musculaires de l'oreille interne sont ainsi le siège de phénomènes actifs directement impliqués dans deux propriétés acoustiques essentielles : la discrimination fréquentielle et les oto-émissions acoustiques.

Par leur contraction, elles modifient localement les propriétés vibratoires de la membrane

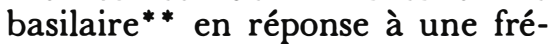
quence donnée. Elles jouent donc un rôle de pré-amplificateur. En pratique, leur atteinte se traduit par la perte de la sélectivité fréquentielle et du pouvoir masquant d'un son par un autre. C'est d'ailleurs ce dont se plaignent de nombreuses personnes âgées incapables de suivre une conversation dans une ambiance bruyante et que peuvent actuellement détecter, dans certains laboratoires spécialisés, des tests audiométriques étudiant les courbes de sélectivité fréquentielle psychoacoustiques (tuning curves).

Par leur contraction encore, les CCE sont à l'origine d'une émission sonore, spontanée ou provoquée par une stimulation acoustique, et susceptible d'être enregistrée par un microphone miniature [2]. L'enregistrement de ces oto-émissions acoustiques paraît donc un moyen relativement simple et particulièrement sensible de détecter une atteinte précoce, infraclinique, des CCE.

Ainsi, la conjonction et l'intensification des efforts cliniques et expérimentaux permet-elle d'espérer dans un avenir proche voir se refermer ce chapitre passionnant mais redoutable de la pathologie iatrogène
Summary

Aminoglycoside-induced ototoxicity results in permanent loss of inner ear function and represents a main issue in the clinical use of these antibiotics in humans. This side effect has stimulated numerous experimental studies in an attempt to clarify the mechanism of ototoxicity. Histological damages affect predominantly the sensory structures. In the cochlea, the lesions progress from the base toward the apex, the outer hair cells being more sentitive to damage than the inner hair cells. Above all, the pattern of cell degeneration in the inner ear appears strikingly different from that observed in the kidney. Electrophysiologic alterations which reflect the functional impairment of the sensory organ precede histological changes. Pharmacokinetics of aminoglycosides in the tissues and fluids of inner ear have been recently clarified. The labyrinthine fluids, perilymph and endolymph, represent an intermediary and obligatory compartment which maintains sustained levels of drug in the vicinity of the susceptible structures. The drug uptake by the tissues seems dose-dependent and manifests a rapid saturation kinetics. The half-life of disappearence varies with the duration of drug treatment and correlates with the development of toxicity, suggesting that noxious effects are related to the penetration of the aminoglycoside into deep(s) compartment(s). Dosage regimen influences the drug uptake as discontinuous administration yields markedly lower tissue concentration than continuous one. At the membrane level, the cochlear and vestibular sensory structures exhibit high-affinity binding sites mainly located at the cell surface. From a clinical point of view, psychoacoustic tuning curves and recording of otoacoustic emissions represent new tools which, by assessing specifically the function of the outer hair cells, may prevent the development of ototoxicity. 